\title{
Vortex corrections to universal scaling of magnetic fluctuations in 2D XY model
}

\author{
Peter C W Holdsworth ${ }^{1}$ 目 and Mauro Sellitto 2 圆 \\ ${ }^{1}$ Laboratoire de Physique, Ecole Normale Supérieure de Lyon, \\ 46 Allée d'Italie, F-69364 Lyon cedex 07, France \\ 2 The Abdus Salam International Centre for Theoretical Physics, \\ Strada Costiera 11, I-34100 Trieste, Italy
}

\begin{abstract}
The vortex contribution to the probability density function of longitudinal magnetization fluctuations is examined in finite 2D XY systems close to the Kosterlitz-Thouless-Berezinskii transition temperature. Within the temperature range studied their relevance is limited to rare fluctuations, where they increase the probability of events exceeding four standard deviations below the mean magnetization.
\end{abstract}

The characterization of fluctuation statistics is a central problem in the study of critical phenomena, as the break down of Landau theory on approaching the critical point, implies a non-Gaussian distribution for order parameter fluctuations [1]. From renormalization group theory it is customary to think of critical phenomena divided into universality classes, characterized by the symmetry group of the order parameter and the spatial dimension. One would therefore expect critical fluctuation statistics to be determined essentially by the set of critical exponents (say $\beta$ and $\nu$ for a regular critical point) which describe the scaling behavior of derivatives of the singular part of the free energy. Evidence from, e.g. the Ising model [2, 3] and Potts models [4] suggests that this is indeed generally the case.

We have, however recently considered an exception to this established phenomenology, the 2D XY model [5, 6]. Here the low temperature phase consists of a line of critical points, with one independent critical exponent $\eta(T)=T / 2 \pi J$ (with $J$ the exchange constant) extending down to zero temperature and separated from the high temperature paramagnetic phase by the Kosterlitz-Thouless-Berezinskii (KTB) phase transition [7, 8, 9]. It is well established that for all temperatures below the KTB transition temperature, $T_{\text {Ктв }}$, renormalisation group flows are to a quadratic effective Hamiltonian [10, 11], with the result that the asymptotic behaviour, in the limit of large system size is perfectly captured by a harmonic model. The advantage of such a simple model is that the probability density function, $P(m)$, for fluctuations of the order parameter, $m$, can be calculated analytically, without using either renormalisation group or the scaling hypothesis. Surprisingly, we find that the form of distribution is independent of $\eta$ along the whole line of critical points. This universal scaling function, plotted as the solid line in Fig. 1, arises when $\sigma P(m)$ is plotted against $\mu=(m-\langle m\rangle) / \sigma$, where $\langle m\rangle$ and $\sigma$ are the mean and standard

$\ddagger$ pcwh@ens-lyon.fr

$\S$ sellitto@ictp.trieste.it 
deviation of the distribution 12, 5, 13]. It is asymmetric, with an exponential tail for fluctuations below the mean and double exponential for fluctuations above the mean.

It has been observed that very similar distributions occur in two and three dimensional Ising models at a temperature $T^{*}(L)$ slightly below, but close to the critical temperature 14, 15]. We have argued that this is a critical phenomenon 114, 16], giving weight to our earlier proposition [12] that many correlated systems, both in and out of equilibrium, can be driven into a state with very similar fluctuations [14, 17], irrespectively of their universality class.

In this Letter we examine the vortex corrections to this universal scaling function as one approaches $T_{\text {Ктв }} \simeq 0.8929(1)$ [18, 19]. We confirm, on the one hand, that below $T_{\text {ктв }}$ the effect of vortex pairs will disappears as the thermodynamic limit is taken, while above $T_{\text {ктв }}$ they become dominant in the same limit. On the other hand, we show that their relevance changes only very slowly with length scale, with the result that finite size corrections to the thermodynamic limit distribution function will be observable over a large range of system sizes.

The 2D XY model is defined by the Hamiltonian

$$
H=-J \sum_{\langle i, j\rangle} \cos \left(\theta_{i}-\theta_{j}\right)
$$

where the angle $\theta_{i}$ gives the orientation of a classical spin vector of unit length, confined to a plane (the sum being over nearest neighbors spins). In the following we consider a square lattice of side $L$ with periodic boundaries, and we set throughout $J / k_{\mathrm{B}}=1$. We define the magnetization $m$ for a single configuration as

$$
m=\frac{1}{N} \sum_{i=1, N} \cos \left(\theta_{i}-\bar{\theta}\right)
$$

where $\bar{\theta}=\tan ^{-1}\left(\sum_{i} \sin \theta_{i} / \sum_{i} \cos \theta_{i}\right)$ is the instantaneous magnetization direction. As the physics of the low temperature phase is perfectly captured by a harmonic, or spin wave Hamiltonian [10, 11], one can, without loss of generality, develop the cosine interaction to second order and neglect the periodicity of $\theta_{i}$. This Hamiltonian is diagonal in reciprocal space and can be solved straightforwardly. In Refs. [5, 6] we find analytically that $\sigma P(m)$, shown as the solid line in Fig. 1, is a universal scaling function, not only of system size, but also of temperature and therefore of critical exponent $\eta$.

We stress that this analytic result is entirely due to harmonic spin waves. At any temperature, corrections to it come from two sources: anharmonic, but analytic terms in the expansion of Eq. (11) and vortex pairs excited as the full periodicity of $\theta_{i}$ becomes important in the region of $T_{\text {ктв }}$ [20]. The former have been discussed in detail in Ref. 15,21]; at fixed temperature, they give small deviations from the universal curve for small system size, $L$. These are finite size corrections to the thermodynamic limit function and disappear with relatively modest increase in the system size.

The two effects can be separated as vortices appear in appreciable numbers in a small range of temperature close to $T_{\text {Ктв }}$ only [22]. In order to study the effect of vortices on the probability density function (PDF) we have carried out extensive Monte Carlo (MC) simulations of the 2D XY model in a small range of temperature around $T_{\text {ктв }}$ and for different system sizes. We typically use systems of linear size $L=16,32,64$. The system was first equilibrated for $10^{5}-10^{6} \mathrm{MC}$ sweeps (MCs), the probability distribution of magnetization was then computed along a trajectory of $10^{7}-10^{8} \mathrm{MCs}$, according to the size of the system. 


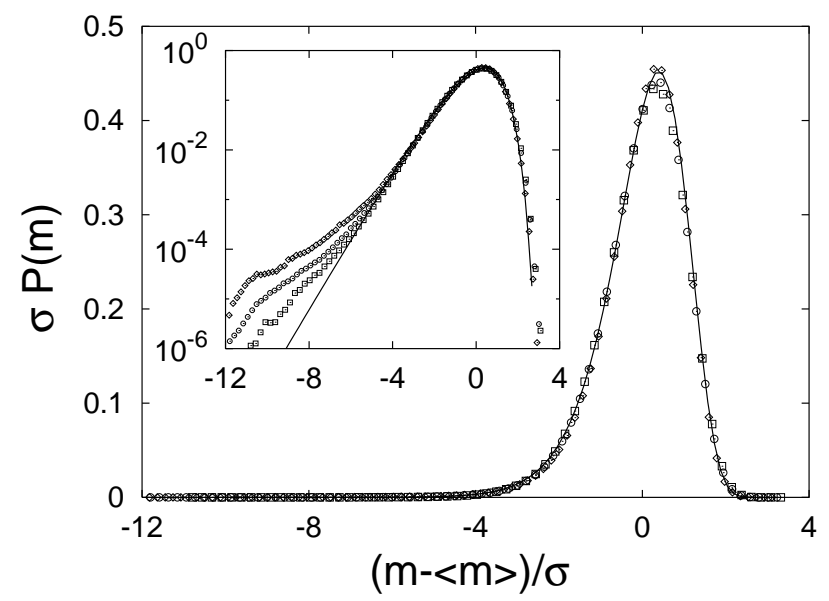

Figure 1. Probability distribution function of magnetization $m$ in a $2 \mathrm{D} \mathrm{XY}$ system of size $L=32$, at temperature $T$ around the $T_{\mathrm{KTB}}$ transition: $T=$ $0.86(\square), 0.89(\circ), 0.92(\diamond)$. In the inset the plot is shown in semi-log scale. The full line represents the exact solution of the harmonic 2D XY model.

In Fig. 1 we show results for a fixed system size $(L=32)$ at several temperatures around $T_{\text {ктв. }}$. First we observe that, when numerical data are plotted in natural units, there is a rather good qualitative agreement with the theoretical curve; a more careful inspection however reveals systematic deviations in the tails: when observed on a semi-logarithmic scale the exponential tail of the distribution changes dramatically, large deviations below the mean magnetization becoming more probable below a characteristic breaking point. This is the vortex contribution. The break point shifts to higher probability with increasing temperature, consistently with the

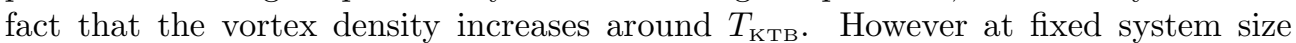
there is no dramatic difference in behaviour on crossing $T_{\mathrm{KTB}}$. The breaking point is a clear signature of two distinct contributions to the PDF [20]: small fluctuations are dominated by spin-waves, while large ones by vortices. It is interesting to note, however that, even at $T_{\text {Ктв }}$, the vortex dominated region is limited to small probabilities and the range $|m-\langle m\rangle|>4 \sigma$, making it difficult to observe. For example Zheng and Trimper in Ref. [15] present numerical results concerning an XY system of size $L=32$ at $T=0.89$; but in their plot deviations to the spin wave result do not appear as their data is limited to the range $|m-\langle m\rangle| \leq 4 \sigma$. Deviations from the spin wave scaling function are also observed in Ref. [13], however the range of temperatures used is outside that for which one expects to see signature of vortices for the probabilities resolved. Note that the best agreement with the data originally published in Ref. [20] is for the highest temperature studied.

Let us now consider how does the vortex contribution change as a function of the system size. In Figs. 2 and 3 we present the PDF at a fixed temperatures, slightly below and above $T_{\text {ктв }}$. In Fig. 2, with $T=0.86$ one can see that, although the effect of changing size is rather small, the deviation from the spin wave result is reduced on increasing $L$, with the vortex dominated region having a system size dependent slope. This is again consistent with a finite size correction to the thermodynamic limit function, although it is clear that much bigger sizes would be required to eliminate the effects of vortices over the range of probabilities shown here. 


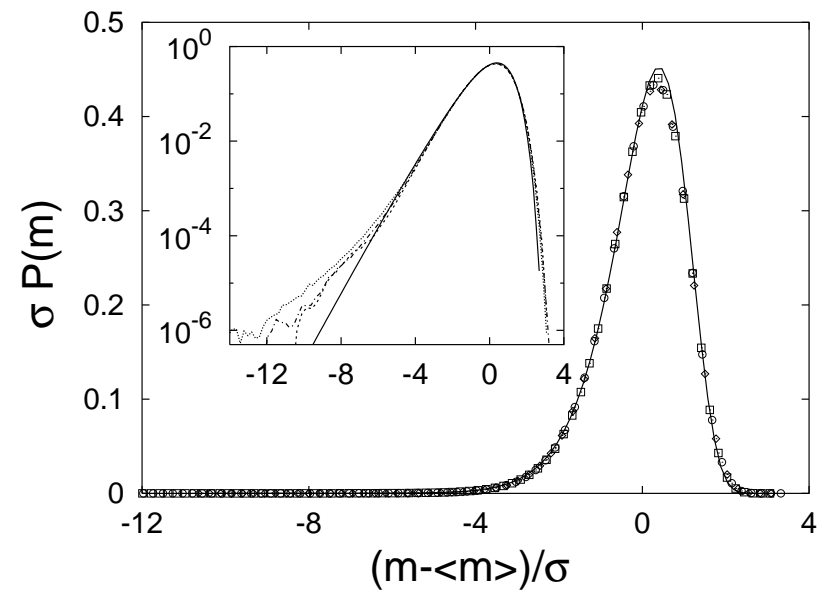

Figure 2. PDF of magnetization $m$ in a $2 \mathrm{D}$ XY system of size $L=$ $16(\square), 32(\circ), 64(\diamond)$, at temperature $T=0.86$ slightly below the $T_{\mathrm{Kтв}}$ transition. In the inset the plot is shown in semi-log scale (dotted, dash-dotted, and dashed lines correspond to $L=16,32,64$ respectively).

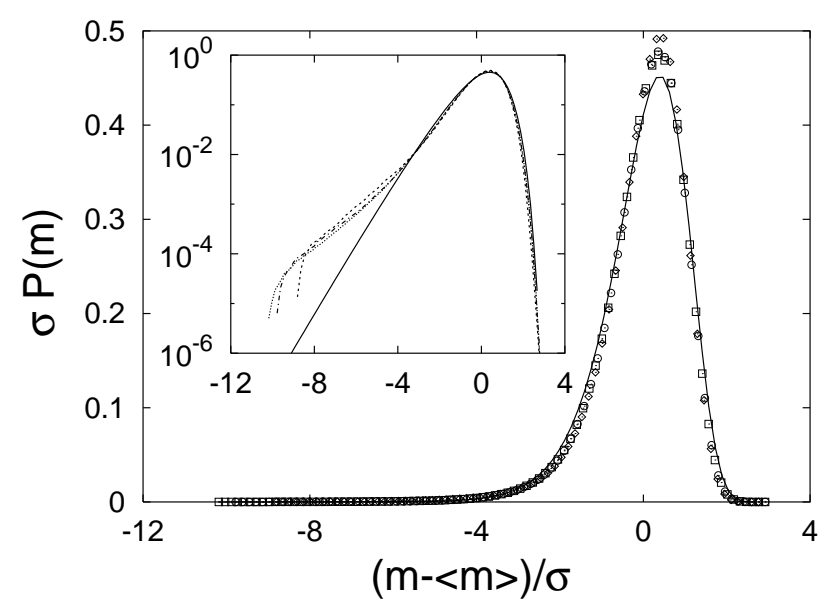

Figure 3. PDF of magnetization $m$ in a $2 \mathrm{D}$ XY system of size $L=$ $16(\square), 32(\circ), 64(\diamond)$, at temperature $T=0.95$ slightly above the $T_{\text {Ктв }}$ transition. In the inset the plot is shown in semi-log scale (dotted, dash-dotted, and dashed lines correspond to $L=16,32,64$ respectively).

¿From Fig. 3 where $T=0.95$, one can see that above $T_{\text {Ктв }}$ just the opposite happens; the slope in the vortex dominated region increases slowly with increasing $L$. The region extends out to a cut off, which decreases with system size. The cut off appears because, with fluctuations of increasing amplitude, the constraint $m \geq 0$ comes into play, limiting the range of possible values of $\mu$. For larger system sizes, or higher temperature the cut off influences the form of the PDF, the topology changes and the system enters the paramagnetic phase through vortex unbinding [20].

Since the spin-spin correlation length, $\xi$ diverges exponentially with the approach 
to $T_{\text {Ктв }}$ from above [8]:

$$
\xi \approx \exp \left(\frac{\pi}{\sqrt{c\left(T-T_{\mathrm{KTB}}\right)}}\right),
$$

$(c \approx 2)$, finite size corrections to $T_{\mathrm{KTB}}$ are logarithmic in $L$ 23, 24]. Defining $T_{C}(L)$ as the temperature where $\xi=L$ gives

$$
T_{C}(L)=T_{\text {Ктв }}+\frac{\pi^{2}}{c(\log L)^{2}} .
$$

At least within finite size scaling terms, this gives an exceptionally large shift. Our results are consistent with this, as at the temperature shown in Fig. 3 the correlation length is in the range of system sizes studied [23. The results beg the question: can one observe scale independence for both the spin wave and the vortex contribution to the PDF, giving a universal two component scaling distribution? Data collapse onto a single curve along a locus of temperatures with varying system size, would be consistent with the notion of the temperature $T^{*}(L)$, introduced in Ref. [23] and put on a more rigorous footing in Ref. [25]. At $T^{*}(L)$, the effective coupling constant, renormalized by the vortex pairs in the system, is independent of length scale, over all lengths up to size $L$ 24. It lies in the interval $T_{\text {ктв }}<T^{*}(L)<T_{C}(L)$ and also scales logarithmically towards $T_{\text {Ктв }}$ with system size. It corresponds to a situation where the vortex-vortex correlations are independent of scale and one would therefore expect two component universality for the PDF at this point. The logarithmic finite size scaling means that this is a difficult question to address quantitatively and we have not attempted to do so here. However, we do show in Fig. 4 data collected at $T=0.93$, for three system sizes, much smaller than the thermodynamic correlation length. We get fairly good data collapse and dependence on $L$ is extremely small over the range studied. There is some deviation from the common curve for $L=64$, and one could clearly obtain better collapse by moving to a smaller temperature. This indicates that at least one locus of points could exist giving data collapse onto a single curve.

In summary, we have presented a quantitative estimation of the vortex contribution to the probability density function of longitudinal fluctuations of magnetization in the 2D XY model, in a band of temperature above and below the Kosterlitz-Thouless-Berezinskii transition temperature. The vortices influence the tail of the distribution for large fluctuations below the mean, giving a break in the exponential tail towards larger probabilities. In the range of parameter space studied, the break is typically for fluctuations of four standard deviations below the mean. Given the expected logarithmic finite size scaling we suggest that this will always be the case, within the physical domain probed numerically or experimentally.

\section{Acknowledgments}

It is a pleasure to thank S.T. Bramwell and B. Portelli for stimulating discussions. MS acknowledges the support of CNRS (contract N. 186078) and the Laboratoire de Physique of ENS-Lyon, where this work was completed. 


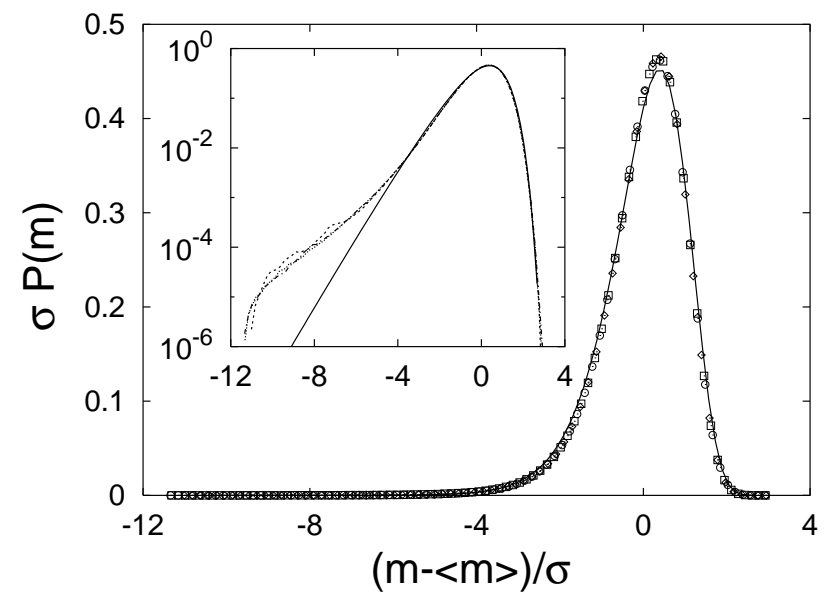

Figure 4. PDF of magnetization $m$ in a $2 \mathrm{D}$ XY system of size $L=$ $16(\square), 32(\circ), 64(\diamond)$, at temperature $T=0.93$. Inset: dotted, dash-dotted, and dashed lines correspond to $L=16,32,64$ respectively.

\section{References}

[1] K.G. Wilson and J. Kogut, Phys. Rep. 1275 (1974).

[2] A.D. Bruce, J. Phys. C 143667 (1981).

[3] K. Binder, in: Computational Methods in Field Theory, H. Gauslever and C. B. Lang eds. (Springer-Verlag, 1992).

[4] R. Botet and M. Ploszajczak, Phys. Rev. E 621825 (2000).

[5] S.T. Bramwell et al., Phys. Rev. E 63041106 (2001).

[6] B. Portelli et al., Phys. Rev. E 64036111 (2001).

[7] J.M. Kosterlitz and D.J. Thouless, J. Phys. C 61181 (1973).

[8] J. M. Kosterlitz, J. Phys. C: Solid State Phys. 71046 (1974).

[9] V.L. Berezinskii, Sov. Phys. JETP 32493 (1971).

[10] J. Villain, J. de Physique 36581 (1975).

[11] J.V. José, L.P. Kadanoff, S. Kirkpatrick and D.R. Nelson, Phys. Rev. B 161217 (1977).

[12] S.T. Bramwell, P.C.W. Holdsworth and J.-F. Pinton, Nature 396552 (1998).

[13] V. Aji and N. Goldenfeld, Phys. Rev. Lett. 861007 (2001).

[14] S.T. Bramwell et al., Phys. Rev. Lett. 843744 (2000).

[15] B. Zheng and S. Trimper, Phys. Rev. Lett. 87188901 (2001).

[16] S.T. Bramwell et al., Phys. Rev. Lett. 87188902 (2001).

[17] R. Labbé, J.F. Pinton and S. Fauve, J. Phys. II 61099 (1996).

[18] R. Gupta et al., Phys. Rev. Lett. 611996 (1988).

[19] M. Hasenbusch and K. Pinn, J.Phys.A 3063 (1997), Physica A 208124 (1994).

[20] P. Archambault, S.T. Bramwell, and P.C.W. Holdsworth_J. Phys. A 308363 (1997).

[21] G. Palma, T. Meyer and R. Labbé, e-print cond-mat/0007289.

[22] H. Weber and H.J. Jensen, Phys. Rev. B 44454 (1991).

[23] S.T. Bramwell and P.C.W. Holdsworth, J. Phys. Condens. Matter 5 L53 (1993).

[24] S.T. Bramwell and P.C.W. Holdsworth, Phys. Rev. B 308811 (1994).

[25] S.G. Chung, Phys. Rev. B 6011761 (1999). 